\title{
The Foundational Significance of Leggett's Non-Local Hidden-Variable Theories
}

the date of receipt and acceptance should be inserted later

\begin{abstract}
Laudisa (Found Phys 38:1110-1132, 2008) claims that experimental research on the class of non-local hidden-variable theories introduced by Leggett is misguided, because these theories are irrelevant for the foundations of quantum mechanics. I show that Laudisa's arguments fail to establish the pessimistic conclusion he draws from them. In particular, it is not the case that Leggett-inspired research is based on a mistaken understanding of Bell's theorem, nor that previous no-hidden-variable theorems already exclude Leggett's models. Finally, I argue that the framework of Bohmian mechanics brings out the importance of Leggett tests, rather than proving their irrelevance, as Laudisa supposes.
\end{abstract}

Keywords Bell's theorem · Bohmian mechanics · Hidden-variable theories · Leggett · Non-locality $\cdot$ Realism

\section{Introduction}

Throughout the debate on the interpretation of quantum mechanics, the "orthodox" view has affirmed the impossibility of completing quantum mechanics by means of hidden variables. One way to understand the significance of John S. Bell's groundbreaking 1964 paper [2] is to say that Bell managed to turn this dogma into an (in principle) empirically testable claim, at least for the class of hidden-variable theories satisfying a certain locality constraint. As is well known, subsequent experiments have vindicated the predictions of quantum mechanics and ruled out local hidden-variable theories. Meanwhile, hidden-variable theories without a locality assumption are not excluded by Bell's theorem.

\section{Egg}

Department of Philosophy, University of Lausanne, CH-1015 Lausanne, Switzerland E-mail: matthias.egg@unil.ch 
This has inspired attempts to go beyond Bell's result by proposing experimental tests also for some classes of non-local hidden-variable theories. The present paper discusses one such attempt, carried out by A. J. Leggett in 2003 [15. Leggett introduced a class of non-local hidden-variable theories which he calls crypto-nonlocal theories and which predict violations of Bell's inequalities, hence they are not excluded by Bell's theorem. However, Leggett showed that they imply a different set of inequalities (henceforth called Leggett's inequalities), which were subsequently tested in experiments 6. 12. The results displayed violations of Leggett's inequalities, in accordance with quantum mechanical predictions. Therefore, Leggett's crypto-nonlocal theories share the fate of local hidden-variable theories: They are ruled out on empirical grounds.

The question now is whether these results tell us anything interesting about the nature of the subatomic world or about the foundations of quantum mechanics. Federico Laudisa [14, p. 1112] vehemently denies that they do. More specifically, Laudisa calls Leggett's approach an "implausible research program" and his theories "totally irrelevant from the viewpoint of the foundations of quantum mechanics". In a nutshell, the arguments leading to this scathing judgment are the following:

1. Leggett's work starts with a mistaken interpretation of Bell's theorem.

2. His theories are already ruled out by well-known no-go theorems of the Gleason-Bell-Kochen-Specker type, so there is no point in testing them experimentally.

3. The consistency of Bohmian mechanics directly refutes Leggett's claims.

The present paper will scrutinize Laudisa's arguments. To set the stage, section 2 gives a brief introduction to Leggett's theories. In section 3, I will argue that Laudisa's first claim, while correct, fails to establish the conclusion he draws from it. Sections 4 and 5 will dispute Laudisa's second claim on theoretical as well as on experimental grounds. Finally, section 6 will show that Laudisa's third claim is not only false, but has things back to front: Far from proving the irrelevance of Leggett-inspired experiments, Bohmian mechanics actually brings out their foundational significance.

\section{Leggett's Non-Local Hidden-Variable Theories}

Leggett considers an EPR/Bohm-type situation with polarization-entangled photon pairs. The measuring apparatus in each wing of the experiment consists of a polarizer (characterized by a transmission axis a for the left wing and $\mathbf{b}$ for the right wing, respectively) and a detector which registers the photon if it is transmitted (rather than absorbed) by the polarizer ${ }^{1}$ Accordingly, the measurement outcomes $A$ (left detector) and $B$ (right detector) can take one of the two values +1 and -1 , depending on whether the detector does or does not register the photon. Leggett now constructs a hidden-variable theory in which each photon pair is characterized by a variable $\lambda$ and a pair of

1 Leggett's results can be proved for general (elliptical) polarization [15. sec. 4], but for the conceptual points I am discussing here, it is sufficient to look at the simpler case of linear polarization. 
polarization directions $\mathbf{u}$ (left photon) and $\mathbf{v}$ (right photon). The interesting difference between such a theory and standard quantum mechanics appears in cases which the latter describes as photon pairs of "indefinite polarization" (e.g., in the so-called singlet state). In contrast to standard quantum mechanics, a Leggett-type theory assumes that each photon pair has a definite polarization, such that the complete ensemble of photon pairs emitted in a series of emission events is a disjoint union of subensembles of definite polarization. In other words, subensembles are characterized by unique values for $\mathbf{u}$ and $\mathbf{v}$. Photon pairs within a given subensemble can, however, have different $\lambda$, where the probability distribution for the $\lambda$ 's is given by $\rho_{\mathbf{u v}}(\lambda)$.

Due to Bell's theorem, such a model can only reproduce the quantum mechanical predictions if it incorporates some kind of non-locality. Within Leggett's theory, the outcomes $A$ and $B$ are therefore allowed to depend not only on the local, but also on the distant parameters:

$$
A=A(\lambda, \mathbf{u}, \mathbf{v}, \mathbf{a}, \mathbf{b}) ; B=B(\lambda, \mathbf{u}, \mathbf{v}, \mathbf{a}, \mathbf{b}) .
$$

If things were kept as general as that, nothing interesting would follow from the assumption of definite polarizations within the subensembles. The crucial constraint is therefore the following assumption, which states that the subensemble averages of $A$ and $B$ (i.e., the averages over all values of $\lambda$ within a given subensemble) depend only on the local variables:

$$
\begin{aligned}
& \bar{A} \doteq \int \rho_{\mathbf{u v}}(\lambda) A(\lambda, \mathbf{u}, \mathbf{v}, \mathbf{a}, \mathbf{b}) d \lambda=2(\mathbf{u} \cdot \mathbf{a})^{2}-1=\bar{A}(\mathbf{u}, \mathbf{a}) \\
& \bar{B} \doteq \int \rho_{\mathbf{u v}}(\lambda) B(\lambda, \mathbf{u}, \mathbf{v}, \mathbf{a}, \mathbf{b}) d \lambda=2(\mathbf{v} \cdot \mathbf{b})^{2}-1=\bar{B}(\mathbf{v}, \mathbf{b}) .
\end{aligned}
$$

Although this is a kind of locality assumption, it is weaker than the assumption from which Bell-type inequalities are derived. Indeed, Leggett [15, sec. 5] shows that a model can satisfy (2) and yet violate Bell's inequalities. Therefore, Leggett-type models are not excluded by Bell tests. This raises the question whether they are compatible with all quantum mechanical predictions. The central result of 15 is that they are not. In analogy with Bell's reasoning, Leggett derives from (2) an inequality which is violated by certain quantum states, thus opening the way to experimental tests of his theories. Performing the relevant experiments, Gröblacher et al. 12 and Branciard et al. 6] confirmed the violations of Leggett's inequalities predicted by quantum mechanics.

No one (to my knowledge) disputes the correctness of these results, but, as we have seen in the introduction, Laudisa claims that they have no foundational significance whatsoever. Before turning to the investigation of Laudisa's arguments, I should mention that Leggett's results have more recently been generalized in several ways [5,8,9]. It might thus seem interesting to evaluate Laudisa's arguments in the context of these newer developments. However, if what I argue below is correct, then this is unnecessary: My basic claim is that even the original results obtained by Leggett, Gröblacher etc. have some foundational significance. But it is obvious that the later, more general results have at least as much foundational significance as the original, 
more limited ones. In other words, if Laudisa's arguments fail with respect to Leggett's original models, they will fail even more dramatically with respect to the more general models developed later. I therefore restrict my discussion to the original Leggett models except for some brief side remarks on later developments (see footnotes 2 and 7 below).

\section{Bell, Leggett, and "Local Realism"}

The bulk of Laudisa's paper [14] is devoted to showing that Leggett and his followers (in particular the authors of [12]) start from a mistaken interpretation of Bell's theorem, which I shall call the $L R$ view. Adherents of this view believe that Bell's theorem is based on two independent assumptions, locality and realism, and that the violations of Bell's inequalities therefore force us to give up at least one of these assumptions. Laudisa is not alone in criticizing this conception 11, 16, 17, and I fully subscribe to the criticism, which may be summarized as follows: Proponents of the LR view either fail to tell us what they mean by "realism" or they use the term to denote something which is demonstrably not an independent assumption of Bell's theorem. If anything, realism is inferred in Bell's derivation, not assumed.

However, from the fact that the LR view is mistaken and that Legett, Gröblacher etc. seem to hold it, it does not follow that research on Leggett's theories is misguided. This would only be the case if the development and the investigation of these theories depended on the LR conception. That there is probably no such dependence can be seen already from the fact that one of the above-mentioned critics of the LR view [11] co-authored one of the papers reporting experimental tests of Leggett's theories 6 .

More generally, the following line of thought shows that one can reject the LR conception and still be interested in Legget-type theories: Once we recognize that Bell did not assume realism for the derivation of his theorem, we see that the LR view is mistaken in suggesting that the violations of Bell's inequalities leave us with a choice to give up either locality or realism. Instead, they simply force us to give up locality. But this leaves open the question whether there is a sense of realism which has to be given up as well. Leggett can therefore be interpreted as investigating theories which give up locality (in accordance with the correct interpretation of Bell's theorem) but hold on to realism in the sense of condition (2).

I do not claim that this is how Leggett himself views the significance of his models. Indeed, some remarks in the first and the last section of [15] indicate his sympathy for the LR inspired view that the ruling out of his non-local realistic theories supports the idea of giving up realism instead of locality. If the LR view is false (as I think it is), then such a conclusion is untenable, because Bell's theorem already rules out all local theories, be they realistic (in whatever sense) or not. But Leggett's problematic conclusion can easily be dissociated from his research program: The investigation of Leggett-type models need not be seen as a test between non-local realism and local nonrealism (whatever that may mean). Instead, we should see it as a test between two kinds of non-local theories, those which respect (2) and those which do not. If one wants to stick to the LR terminology, one may call these theories 
non-local realistic and non-local non-realistic, respectively, but these labels are not very helpful $]^{2}$ except perhaps to drive home the point that there is a sense in which the investigation of non-local realistic theories, as proposed by Leggett, is perfectly acceptable even for the opponent of the LR view.

\section{Leggett's Models and the Kochen-Specker Theorem}

Laudisa's second reason to question the significance of testing Leggett-type theories is that their realism assumption (which he denotes by REALISM G\&AL $_{\text {, }}$ the subscript referring to Gröblacher et al.) already dooms them to failure in the light of previous no-hidden-variable theorems.

If REALISM G\&AL were an independent assumption of any hidden variable theory, Gleason-Bell-Kochen \& Specker would have already proved their incompatibility with quantum mechanics needless of any locality requirement. [14, pp. 1122-1123]

Unfortunately, Laudisa here falls into the very trap that often undermines the intelligibility of the LR view: the use of an insufficiently precise notion of "realism". The conception of realism which he attributes to Gröblacher et al. includes non-contextuality in the following sense: "The physical systems under scrutiny are endowed with preexisting properties that do not depend essentially on the measurement interactions the systems themselves may undergo" [14, p. 1113]. If this is understood such that individual measurement outcomes are independent of what other measurements are made simultaneously, then it is indeed a form of non-contextuality which is incompatible with quantum mechanics, as shown by the (Gleason-Bell-)Kochen-Specker theorem ${ }^{3}$ But do Gröblacher et al. really assume this kind of non-contextuality? At first sight, it seems that they do, by assuming that "all measurement outcomes are determined by pre-existing properties of particles independent of the measurement (realism)" [12, p. 872]. But just two sentences later they add that "measurement outcomes may very well depend on parameters in space-like separated regions". And if one looks what condition of non-contextuality they actually use in their derivation, then it turns out that it is non-contextuality on the subensemble level, as spelled out in (2). This is compatible with a failure of non-contextuality on the level of individual measurements, as expressed in (1). Since the Kochen-Specker theorem only rules out theories which are non-contextual in this latter sense, it has nothing to say about the Leggetttype theories investigated by Gröblacher et al. In fact, it is rather surprising that Laudisa should have missed the importance of distinguishing the individual level from the (sub-)ensemble level when discussing non-contextuality,

2 One of the reasons why the LR terminology is unhelpful is that it obscures the fact that (2) is itself a kind of locality condition. This is reflected in the more appropriate terminology introduced by Colbeck and Renner 8, who classify Leggett's models as models for which the hidden variables have both a local and a global part, as opposed to entirely nonlocal models. This distinction forms the basis of Colbeck's and Renner's generalization of Leggett's results: As they demonstrate, all models having a nontrivial local part (not just those which satisfy the particular condition (2)) are incompatible with quantum mechanical predictions.

For a concise presentation of the essential part of the theorem, see [3, sec. 5]. 
because in an earlier paper [13, pp. 492-493] he himself used precisely this distinction to show why neither Bell's hypothetical local hidden-variable theory 2 nor Bohm's theory 4 fall prey to the existing no-go theorems for non-contextual hidden-variable theories.

\section{The Significance of Experimental Tests}

The previous section has shown that the theorems cited by Laudisa do not suffice to establish the incompatibility between Leggett's models and quantum mechanics; the incompatibility only manifests itself through Leggett's inequality and its violation by quantum mechanical predictions. These conflicting predictions can then be tested in experiments. But again, Laudisa [14, p. 1123] disputes the significance of such tests. Immediately following the sentence quoted above, he writes:

But, as Bell showed, there is little significance in testing against quantum theory a theory (be it local or non-local) that is supposed to satisfy a condition that we already know quantum mechanics cannot possibly and reasonably satisfy.

I am not convinced by this reasoning. Indeed, the best counterexamples to this claim are Bell's inequalities themselves. The fact that these inequalities are violated by the quantum mechanical predictions shows that quantum mechanics "cannot possibly and reasonably satisfy" the conditions assumed for their derivation. Should we therefore conclude that Aspect's experiments (to name just the most famous example) are of "little significance"? This would amount to a dubious a priori commitment to the truth of quantum mechanical predictions in domains where quantum mechanics has not yet been tested.

I suspect that Laudisa fails to appreciate the force of this counterexample because he does not properly distinguish between theoretical and experimental aspects of Bell's theorem. This can be seen by analyzing the following extract from what Laudisa offers as the logical reconstruction of the BellClauser-Horne argument. (The complete argument includes six steps, but we only need to look at steps 2 to 4 ; here "QM" stands for "the assumption of the validity of the statistical predictions of quantum mechanics" [14, p. 1124], while "BI" denotes Bell's inequalities.)

2. $\mathrm{QM} \rightarrow \neg \mathrm{BI} \quad$ [Experimental fact]

3. QM [Assumption]

4. $\neg \mathrm{BI}[2,3$ Modus ponens $]$

[14, p. 1127; square brackets in the original]

This way of putting things strikes me as thoroughly confused. First of all, the conditional in step 2 is not an experimental fact. That quantum mechanical predictions violate Bell's inequalities can be derived (and is in fact derived by Clauser et al. 7]) on a purely theoretical basis, without the need for any experiment. What is an experimental fact is the observed violation of Bell's inequalities, for example in Aspect's experiment [1, as expressed in step 4. By presenting this step as the result of a modus ponens, Laudisa creates 
the false impression that one needs to assume the truth of QM (step 3) in order to conclude that Bell's inequalities are violated. Now it is true that, as a matter of practical fact, a commitment to some well-established parts of QM (concerning, for example, the production of entangled particle pairs) is involved in performing a Bell-type experiment. And it is also true that, historically, most performers of Bell tests expected all quantum mechanical predictions to turn out correct ${ }^{4}$ But no assumption of the correctness of QM (in the sense that would logically imply violations of Bell's inequalities, as in Laudisa's step 4) is involved in performing these experiments. The very point of carrying out a Bell-test is to treat the correctness of theoretical predictions regarding BI as an open question, to be decided by experiment, rather than to be derived from our preconceived assumptions. This is how the result $\neg \mathrm{BI}$ is obtained.

\section{Leggett-Type Theories and Bohmian Mechanics}

As a final argument against Leggett's approach, Laudisa [14, p. 1129] cites the consistency of Bohmian mechanics and claims that this "directly refutes the claims of Leggett and followers". His idea seems to be that Bohmian mechanics, by being a hidden-variable theory which is consistent with all quantum mechanical predictions (and all empirical data), somehow invalidates Leggett's claim that the hidden-variable theories he investigates are in conflict with some quantum mechanical predictions (and with some possible empirical data). But of course, Bohmian mechanics could only serve as a counterexample in this sense if it belonged to the class of Leggett-type theories. Laudisa seems to believe that it does, as he claims that "Bohmian mechanics satisfies REALISM G\&AL [ibid.], but this is clearly false. ${ }^{5}$ As described above, an essential component of REALISM G\&AL $_{\text {is }}$ ise commitment to a definite polarization of each photon, giving rise to the subensembles underlying (2). Bohmian mechanics, by contrast, does not regard polarization as a property of individual particles, and it is therefore not a REALISTIC G\&AL theory. Put in terms of hidden variables, the difference between Bohmian mechanics and a Leggett-type theory is that the two theories are committed to different hidden variables: position in the first case, polarization in the second.

While it is thus unjustified to view Bohmian mechanics as directly refuting Leggett's approach, a certain tension between the two cannot be denied. In particular, from a Bohmian perspective, it amounts to "naive realism" [10] to treat as real any property other than position. But it is at least conceivable that the Bohmian perspective - whithin which position takes priority over all other properties - is not the only possible way to construct a hidden-variable

4 A relevant counterexample is John Clauser, who seems to have been genuinely convinced that Bell tests were going to disprove quantum mechanics (see [18, p. 160].

5 It should be false even by Laudisa's own lights: Since he is convinced that REALISM $_{\mathrm{G} \& \mathrm{AL}}$ is an unreasonable assumption [14, p. 1128], satisfying it would make Bohmian mechanics an unreasonable theory, which is certainly not what he wants to claim. 
theory. More specifically, if we speak about photons (which not even Bohmian mechanics describes as continuously localized particles), there is no a priori reason why we should regard position as a more fundamental property than polarization. Leggett's proposal can then be seen as an attempt to explore the empirical consequences of an alternative to the Bohmian perspective. Under this interpretation, Bohmian mechanics actually brings out the relevance of Leggett-inspired research, instead of making it obsolete, as Laudisa supposes.

Let me explain this by once more highlighting the parallel between Leggett's and Bell's inequalities. A frequently heard complaint about Bohmian mechanics is that it is non-local. The correct response to this is to refer to the experimental violations of Bell's inequalities, which show that non-locality is not a peculiarity of Bohmian mechanics, but an experimental fact. In a parallel fashion, experimental violations of Leggett's inequalities furnish a reply to another complaint that is sometimes made against Bohmian mechanics, namely its non-realism with respect to all properties except position ${ }^{6}$ Experimental tests of Leggett-type models support the Bohmian approach by demonstrating that a realism about polarization, even in the modest sense of (2), is in conflict with empirical data 7 The Bohmian should therefore not join Laudisa in denouncing Leggett's research program as irrelevant, but should rather welcome it as significantly supporting his own position, by showing that non-realism about the polarization of individual photons is not just a theoretical postulate, but an experimental fact.

Acknowledgements I am grateful to the audiences at the Workshops The Metaphysics of Contemporary Physics (Lausanne, May 2012) and Causality and Physics (Munich, August 2012) for helpful discussions. I also wish to thank Detlef Dürr and two anonymous referees for insightful comments.

\section{References}

1. Aspect, A., Dalibard, J., Roger, G.: Experimental test of Bell's inequalities using time-varying analyzers. Physical Review Letters 49, 1804-1807 (1982)

2. Bell, J.S.: On the Einstein-Podolsky-Rosen paradox. Physics 1, 195-200 (1964)

3. Bell, J.S.: On the problem of hidden variables in quantum mechanics. Reviews of Modern Physics 38, 447-452 (1966)

4. Bohm, D.: A suggested interpretation of the quantum theory in terms of 'hidden' variables. I and II. Physical Review 85, 166-193 (1952)

5. Branciard, C., Brunner, N., Gisin, N., Kurtsiefer, C., Lamas-Linares, A., Ling, A., Scarani, V.: Testing quantum correlations versus single-particle properties within Legget's model and beyond. Nature Physics 4, 681-685 (2008)

6 Daumer et al. [10, 382] even call this a "frequent complaint", and their paper is a spirited reply to it. But in contrast to my argument from experimental violations of Leggett's inequalities, their reply presupposes the Bohmian perspective and the privileged role it assigns to positions.

7 The work of Colbeck and Renner 8 licenses an even stronger conclusion, by no longer depending on the specific form of the constraint 22 (see footnote2 above), and it therefore strengthens the kind of support for Bohmian mechanics described here. However, in a more recent paper, Colbeck and Renner 9, p. 13] criticize Bohmian mechanics for being incompatible with a very natural freedom of choice assumption. It seems to me that Bohmians would insist on a different notion of free choice than the one employed by Colbeck and Renner, but this issue is beyond the scope of this paper. 
6. Branciard, C., Ling, A., Gisin, N., Kurtsiefer, C., Lamas-Linares, A., Scarani V.: Experimental falsification of Leggett's nonlocal variable model. Physical Review Letters 99, 210407 (2007)

7. Clauser, J.F., Horne, M.A., Shimony, A., Holt, R.A.: Proposed experiment to test local hidden-variable theories. Physical Review Letters 23, 880-884 (1969)

8. Colbeck, R., Renner, R.: Hidden variable models for quantum theory cannot have any local part. Physical Review Letters 101, 050403 (2008)

9. Colbeck, R., Renner, R.: The completeness of quantum theory for predicting measurement outcomes (2012). arXiv:1208.4123v1

10. Daumer, M., Dürr, D., Goldstein, S., Zanghì, N.: Naive realism about operators. Erkenntnis 45, 379-397 (1997)

11. Gisin, N.: Non-realism: Deep thought or a soft option? Foundations of Physics 42, 80-85 (2012)

12. Gröblacher, S., Paterek, T., Kaltenbaek, R., Brukner, Č., Żukowski, M., Aspelmeyer, M., Zeilinger, A.: An experimental test of non-local realism. Nature 446, 871-875 (2007)

13. Laudisa, F.: Contextualism and nonlocality in the algebra of EPR observables. Philosophy of Science 64, 478-496 (1997)

14. Laudisa, F.: Non-local realistic theories and the scope of the Bell theorem. Foundations of Physics 38, 1110-1132 (2008)

15. Leggett, A.J.: Nonlocal hidden-variable theories and quantum mechanics: An incompatibility theorem. Foundations of Physics 33, 1469-1493 (2003)

16. Maudlin, T.: Space-time in the quantum world. In: J.T. Cushing, A. Fine, S. Goldstein (eds.) Bohmian Mechanics and Quantum Theory: An Appraisal, Boston Studies in the Philosophy of Science, vol. 184, pp. 285-307. Kluwer Academic Publishers, Dordrecht (1996)

17. Norsen, T.: Against 'realism'. Foundations of Physics 37, 311-340 (2007)

18. Whitaker, A.: The New Quantum Age: From Bell's Theorem to Quantum Computation and Teleportation. Oxford University Press, Oxford (2012) 